\title{
Parteienprofile: Ist die CDU pensionsreif?
}

von Tilman Mayer

Seit ihrer Gründung im Jahr 1945 hat sich die CDU zu einer staatstragenden Volkspartei der Bundesrepublik Deutschland entwickelt. Dieser Beitrag sucht diese Evolution nachzuzeichnen, spezifische Erfolgsdeterminanten zu benennen sowie die Genese der gegenwärtigen Form und Funktion der Partei zu analysieren. Die Aufmerksamkeit richtet sich dabei neben ideen- und machtpolitischen Fragen auf eine Reihe einzelner Politikbereiche, die Rolle der CDU in der deutschen Parteienlandschaft, den organisationspolitischen Umgang mit der Wiedervereinigung und der bayerischen Schwesterpartei CSU sowie schließlich auf aktuelle Fragen der Innen-, Europa- und Außenpolitik. Im Fazit steht, dass die CDU ihre gegenwärtige Lethargie durch programmatische, mobilisierende Aussagen bewältigen muss, um nicht als pensionsreif gebrandmarkt zu werden.

Since its foundation in 1945, the CDU has become a core element of the (West) German party system. This contribution traces said development, identifies specific determinants of success and analyses the party's current form and function. In addition to questions of ideology and power, attention is devoted to a number of factors including programmatic aspects in several policy areas, the position of the CDU in the German party landscape, its reaction to German unification and its strategy towards its Bavarian counterpart, the CSU. This is complemented by a discussion of current domestic, European and foreign policy issues. It is concluded that the CDU needs to break out of its present state of lethargy by voicing programmatic claims and by mobilising its supporters in order to avoid being perceived as "ready for retirement".

\section{Was zusammengehört}

Die 1945 gegründete Christlich Demokratische Union (CDU) hat jenes Lebensalter überschritten, das den Bürger im Regelfall in den Ruhestand führt. Ist es Zeit, die CDU in Pension zu schicken? Hat sich diese große Partei überlebt?

Dieses Bild einer organischen Entwicklung trägt heutzutage nicht sehr weit, zumal ein solcher Befund nicht ganz korrekt formuliert wäre: Die CDU bezeichnete sich nie als Partei, sondern wurde als Union gegründet. Dies war von Beginn an eine sehr moderne Selbstdefinition, da sie eine Einheit ohne organische Einheitlichkeit suggeriert. Eher ist die CDU ein Projekt, ein sehr spezifisches zudem. Union - wer sich so nennt, der hat den Anspruch, mehr zu sein als eine Partei. Im Übrigen formulierte auch die konkurrierende Sozialistische Einheits- 
partei Deutschlands (SED) ab November 1945 den Anspruch, übergreifend Interessen organisieren zu können. Allerdings blieb es dort bei dem Wunsch, im Fall der CDU hingegen ist der Anspruch bis heute prägend.

Die CDU war 1945 eine moderne Erfindung. Die Partei tauchte überraschend auf, sie passte aber machtstrategisch wie programmatisch in die politische Arena. Schnell wurde sie in der Nachkriegszeit zum Magneten für ehrgeizige Politiker, die sich auch andere Formationen vorstellen konnten, nicht zuletzt Konrad Adenauer. Der Clou der Parteigründung bestand darin, etwas zusammenzubringen, was zuvor nicht zusammenführbar schien.

Der Unionsgedanke war auch eine Antwort auf den untergegangenen Nationalsozialismus, der beansprucht hatte, soziale Gegensätze überwinden zu können. Doch die ,Volksgemeinschaft“ blieb ebenso Fiktion wie die ,klassenlose Gesellschaft" der SED. Dagegen bemühten sich die Vertreter unterschiedlicher sozialer Gruppen, Arbeitgeber wie Arbeitnehmer, den Gedanken des Zusammenhalts in Gestalt einer gemeinsamen Union zu organisieren. Bedenkt man die konfessionelle Spaltung der deutschen Gesellschaft, die im Bismarckreich die Nationalstaatsbildung im Innern kulturell belastete, offenbart sich der Fortschritt, der durch ein konfessionsübergreifendes Bündnis nach 1945 erreicht werden konnte. Die konfessionellen Kräfte der Beharrung wurden auch hier nur durch die Erfahrung von Repression, Verfolgung und Widerstand im Nationalsozialismus überwunden, die die Gemeinsamkeiten stärker ins Bewusstsein der konfessionell Denkenden rückte.

Ein dritter Angelpunkt des neuen Zusammenhalts war - neben dem sozialen und dem konfessionellen - nach 1945 nötig, um Geltung zu erlangen. Die Christlich Demokratische Union Deutschlands, CDUD, wie sie in der SBZ und in OstBerlin genannt wurde, sorgte für eine nationale Union, d. h. den Zusammenhalt von Ost und West, der 1945 und in den Jahren danach aufgrund außenpolitischer Konstellationen verloren ging. Aber allein den Gedanken auch in der Parteikonstruktion festzuhalten und zum Ausdruck zu bringen, legitimierte den Unionscharakter dauerhaft. Die CDU wurde also von Beginn an und durch ihren Namen als eine gesamtdeutsche Partei formiert. So könnte man sich die Partei als nahezu progressiv vorstellen, denn sie musste ihrem Anspruch immer wieder genügen, den sozialen Unionscharakter in der Gesellschaft umzusetzen, den religiösen Zusammenhalt auch durch die Benennung von inkorporierten Konfessionen sichtbar zu machen und den Gedanken an die Einheit der Nation wach zu halten. Die Gefahr, dass die einzelnen Ansprüche durch Überbetonung zu einer einseitigen Entwicklung führen könnten, war potentiell gegeben. 


\section{Verzicht auf Restauration}

Das Verdienst der Gründung einer christdemokratischen Union liegt im Rahmen der politischen Kultur Deutschlands gerade darin, dass kein monokonfessionelles „Zentrum“ mehr, keine konfessionell-fundamentale Position oder Disposition mehr eingenommen wurde. Es kam in der Nachkriegszeit eben nicht zu einer Restauration dessen, was sich in der Weimarer Republik als zu schwach erwies, um die Republik nachhaltig zu stützen oder gar zu steuern. Der schon zur Weimarer Zeit entfaltete Gedanke eines transkonfessionellen Bündnisses, zunächst von den christlichen Gewerkschaften entwickelt, entfaltete erst nach 1945 seine gesamte Energie und strukturierte damit die (wenn auch vorerst nur westdeutsche) Gesellschaft von Grund auf neu. ${ }^{1}$

Eine derart breit angelegte Formation hatte den großen Vorteil, für eine Kohäsion der Gesellschaft zu sorgen, die in der Nachkriegskonstellation ihrerseits parzelliert und neu zusammengesetzt wurde. Eine demoralisierte Gesellschaft, die in vielen katastrophal zerstörten Städten mühsam einen Neuanfang suchen musste, die eine immens große Zahl von Vertriebenen aufzunehmen hatte, war darauf angewiesen, dass nicht auch noch das Parteiwesen diese Unterschiede betonte. So bildete die neu entstandene Union eine Integrationsformation, die zur Festigung des jungen Gemeinwesens einen starken Beitrag leistete und als Neuansatz dem Aufbruch - dieser ist zu betonen, nicht den immer wieder behaupteten Muff der 1950er Jahre ${ }^{2}$ - einer Nachkriegsgesellschaft eher entsprach als die Neugründung von weltanschaulich orientierten Parteien. Vergegenwärtigt man sich noch mal den Kulturkampf, den das Deutsche Reich Bismarcks sich mit dem Katholizismus leistete, wird noch deutlicher, welche deeskalierende Wirkung das programmatische Unionskonzept für die damalige Gesellschaft hatte - zumal im Kalten Krieg, als der außenpolitische Druck der jungen Bundesrepublik stark zusetzte.

\section{Die Rolle von Ideenkreisen}

Es wäre allerdings falsch, zu meinen, ideologische Konstellationen hätten keine Rolle mehr gespielt. Von solchen Dispositionen ist im Gegenteil unbedingt aus-

1 Buchstab, G. (Hrsg.): „Es musste alles neu gemacht werden“ - Die Protokolle des CDUBundesvorstandes 1950-1953, Stuttgart, 1986.

2 Hans-Peter Schwarz sprach von einer ,Epoche aufregender Modernisierung“, vgl. auch Mayer, T. (Hrsg.): Medienmacht und Öffentlichkeit in der Ära Adenauer, Rhöndorfer Gespräche, Bd. 23, Bonn, 2009. 
zugehen, ${ }^{3}$ auch hier kann man allerdings den Unionscharakter herausstreichen. Im christlich-demokratischen Gedankenüberbau werden ideologische Strömungen aus dem Konservatismus, dem christlich-sozialen Gedanken, dem liberalen Spektrum und der nationalen Identität zusammengefasst. Das Moment des Christlichen mischt sich mit diesen Strömungen. Sie sind präsent, aber nie dominant. In unterschiedlichen Zeiten, im 20. wie 21. Jahrhundert, werden sie unterschiedlich stark sichtbar oder personifiziert vertreten. Aber es zählt zum Wesen dieser Union, dass auch sehr exponierte Vertreter der unterschiedlichen Flügel das Gesamtbild der Partei nicht allein bestimmen (konnten).

Deswegen ist es auch grundlegend falsch, allein auf das sogenannte ,hohe C“ als Verkörperung der Identität der Union zu setzen - was ja vom Namen her naheliegt. Die CDU ist kein Zentrum, sie ist keine klerikale Partei. Es wirkt öfters peinlich, wenn man auf der Suche nach ihrer Identität hilflos auf das „C“ starrt und meint, von dort käme das Heil. ${ }^{4}$ Derart gedankenarme Behelfslösungen tragen nicht weit und nehmen Personen in die Pflicht, die mit der CDU als politische Formation nicht ohne weiteres in Verbindung gebracht werden können. Der Hilferuf also, die Bischöfe mögen doch bitte darüber aufklären, was das ,hohe $\mathrm{C}^{\text {" }}$ bedeutet, bleibt eine hilflose Idee, die in der Geschichte der CDU jedoch schon mehrfach formuliert wurde. ${ }^{5}$ Es kann nicht oft genug wiederholt werden, dass die CDU, aus christlich-kirchlicher Perspektive gesehen, eine Formation von „Laien“ ist, die mithin eigenverantwortlich, als christliche Bürger, handeln, sich einem mehr oder weniger christlichen Grundverständnis verpflichtet fühlen, aber sicherlich nicht im Sinne einer klerikalen Partei der Autorität eines Bischofs bedürfen, um zu wissen, was sie wollen sollen. Wenn sich die Bischöfe ihrerseits berufen fühlen, der CDU ungefragt Ratschläge zu erteilen, weil sie eine christliche Partei ist, geschieht dies aus einem unpolitischen, ja naiven Impuls heraus zur Freude der Gegner der Union. Insofern kann man sehen, dass der Zusammenhang von christlicher Partei und katholischer oder evangelischer Kirche nicht einfach zu erklären ist.

Für die Gegner der CDU sieht es ganz anders aus. Sie können je nach Lage einerseits die klerikalen, andererseits die nicht-christlichen Politikansätze der je-

3 Scherer, P.: Jenseits von Links und Rechts: Spielt Ideologie für Parteien und Wähler keine Rolle mehr?, in: Bytzek, E./Roßteutscher, S. (Hrsg): Der unbekannte Wähler? Mythen und Fakten über das Wahlverhalten der Deutschen, Frankfurt, 2011, 23-41.

4 Anderer Ansicht ist Kauder, V.: Das C ist für uns Programm, in: FAZ, 9. Juli 2008, 7.

5 Vgl. dazu die verdienstvollen Werke von Rudolf Uertz, der diesen wichtigen Gedanken immer wieder versucht hat aufklärend zu vermitteln. 
weiligen Regierung herausstellen, um dem christlichen Wähler zu imponieren. Allerdings bleibt der Anspruch der CDU, christlich denkende Bürger anzusprechen eine auch machtstrategisch günstige Disposition in einer Gesellschaft, die christlich fundiert ist und kulturell christlich fundiert bleibt - selbst in der Distanz zu einem stärker praktisch gelebten, praktizierten Christentum. Christlichkeit ist für die CDU als christliche Partei, um es paradox auszudrücken, nicht alles. Sie deckt nicht das ganze Spektrum an Identitäten ab, die in der Union Entfaltung suchen.

Die erwähnten liberalen, konservativen, sozialen und nationalen Elemente haben innerhalb des Unionsbaus ihre Eigenberechtigung. In dem Maße, in dem sie sich unter dem christlich-demokratischen Dach versammeln können und sich integrativ äußern, trägt dies erheblich zur Festigkeit dieses Baus bei. Insofern ist es vollkommen legitim, diese ideologischen Strömungen zu identifizieren. Umgekehrt stellt es einen Verlust dar, wenn sie nicht mehr oder nur noch in geringem Maß vorhanden sind. Nochmals: Das Verdienst der CDU in der politischen Kultur der Bundesrepublik (auch nach der Wiedervereinigung) liegt in diesem integrativen Moment - für die Gesellschaft wie die Partei. Die einzelnen Strömungen sollten auch eine gewisse Sichtbarkeit behalten, denn wenn sie vollständig verblassen sollten, würde dies der politischen Diversität und damit dem Sammlungscharakter der Partei schaden. Nach französischem Muster kann die CDU nämlich durchaus als Sammlung bezeichnet werden, als Rassemblement pour la République Allemande, das die unterschiedlichen ideologischen Ansätze zur Melodie eines Schlagers vereint.

Jeder, der dagegen die erwähnten Ideenkreise in reiner Form erleben möchte, hat in den Unionsparteien einen Gegner. Sie müssen integriert werden. Insofern ist die Alleinvertretung spezifisch liberaler, sozialdemokratischer, sozialistischer und anderweitiger Ideenkreise in Reinkultur mit dem Unionscharakter nicht kompatibel. Die Attraktivität weltanschaulicher Profile besteht allerdings fort. Daher sind die Unionsparteien ihrerseits durch diese Attraktivität herausgefordert. Auch hier wäre eine Reduktion der Programmatik der CDU auf das ,hohe C“" taktisch wie strategisch unklug und entspräche kaum mehr dem Lebensgefühl ihrer Mitglieder und Anhänger.

Das ideenpolitische Arsenal der CDU ist in der Summe mehr wert, als jeder Versuch der einzelnen Teile des ideologischen Spektrums, ihre Interessen eigenständig in die Politik hineinzutragen. Das politische Paket-Angebot der CDU, in dem viele Ideenkreise verpackt sind, kommt auch dem christlichen Moment zugute, das, auf sich gestellt, in einer säkularen Gesellschaft nur geringe Ein- 
flussmöglichkeiten hätte. Insofern sind alle fundamentalistischen Ansätze und Anläufe rückständig im Vergleich zum Unionskonzept, das in einem Art „Arche Noah"-Ansatz vieles mittragen kann, was sonst untergehen würde bzw. längst untergegangen wäre. Natürlich ist dieser Ansatz nicht für alle Zeiten garantiert und kann durch die Unfähigkeit Einzelner beschädigt werden. Insofern vermag von Zeit zu Zeit die Eitelkeit bestimmter Individuen dem Gesamtinteresse der Partei entgegenstehen. ${ }^{6}$

Die Partei muss auch aushalten, sich einerseits nicht zu spezifisch zu positionieren oder sich zu sehr in den Dienst einzelner Interessen zu stellen. Sie darf sich andererseits auch nicht zu allgemein und interessenlos geben. Beides würde zum Verlust von Zustimmung führen. Die Kunst der Führung einer derartigen Partei liegt auf der Hand.

\section{Führungsposition}

Eine isolierte Darstellung kann einer Charakterisierung der CDU jedoch kaum genügen. Die Partei steht nicht für sich allein. Im Vergleich zu allen anderen Parteien der Bundesrepublik bestand ihre Aufgabe zu jeder Zeit darin, die Fähigkeit zu besitzen, eine number one-Position einzunehmen. Die CDU war so auch immer eine Partei des leadership. In der Geschichte der Bundesrepublik wurde sie in dieser Position unersetzbar. Sie hat das Staatsschiff auch in Krisenzeiten gesteuert, war in unterschiedlichen Konstellationen koalitionsbildend und v.a. stets der entscheidende Faktor, der machtpolitische „Treiber“ innerhalb dieser Koalitionen. Zwar war die CDU nicht dominant wie die italienische Democrazia Cristiana, doch sie fungierte als eine entscheidende Kraft, mit der man Politik betreiben konnte (und in der Mehrzahl der Fälle auch musste). Im Unterschied zu Italien hat die CDU dabei nicht an Substanz verloren oder ihre Integrität aufgegeben. Zudem wurde sie durch den Zerfall des Gegners - des Kommunismus kaum beschädigt, sondern blieb eine große Partei, die die Zeitenwende zwar das ist das große Thema von Beobachtern, die den Niedergang konstatieren ${ }^{7}$ - in

$6 \mathrm{Ob}$ dies bereits im Falle des sich bekanntermaßen als „Querdenker“ gerierenden Herz-Jesu-Politikers Heiner Geißler der Fall ist, der Bücher mit dem Titel schreibt: „Was würde Jesus heute sagen? Die politische Botschaft des Evangeliums“, muss hier nicht entschieden werden. Dagegen hat die rhetorische Leistung eines Heiner Geißler als CDU-Generalsekretär in den 1980er und 1990er Jahren dazu geführt, dass sich die CDU gegenüber der SPD auch in Arbeiterkreisen behaupten konnte. Ähnliches gilt für Norbert Blüm, der sich gelegentlich zurückmeldet: Mehr Obst, weniger Äpfel? Die CDU in der liberalen Falle oder Wider den Imperialismus der Verwirtschaftung des Lebens, in: FAZ, 5. September 2001, 12.

7 Probst, L.: Der Abstieg der Volksparteien. Die neue Beweglichkeit im Fünfparteiensystem, in: Kommune, 3 (2010), 18-21; Weßels, B.: Was ist dran an der These vom Ende der Sozialdemokratie?, in: Friedrich-Ebert-Stiftung, Internationale Politikanalyse, Berlin, 2010; Langguth, G.: Volksparteien ohne Volk? 
kleinerer Gestalt, aber durchaus intakt überdauerte. Der entscheidende Punkt ist, dass die CDU - mehr oder weniger ähnlich der SPD - Verantwortung im umfassenden Sinne übernimmt, mithin nicht nur für kleine Politikanteile in Anspruch genommen wird, wie dies bei den Grünen, den Liberalen und ggf. auch anderen Parteien der Fall ist. Die CDU zählt mit der SPD zu den Parteien, die nach wie vor die Fähigkeit besitzen, Direktmandate zu erobern. Dieses Qualitätsmoment kommt nahezu keiner anderen Partei zu - kleinere Veränderungen in jüngster Zeit eingerechnet.

Ein weiteres positives Merkmal liegt darin, dass die leadership-Funktion der CDU im exekutiven Bereich ein (mit der SPD geteiltes) Alleinstellungsmerkmal darstellt. Selbst auf der Ebene der Oberbürgermeister großer Städte bildet sich diese Verantwortungsstruktur deutlich ab. Man kann daraus deskriptiv schlussfolgern, dass die Bevölkerung über viele Jahrzehnte der Meinung blieb, entscheidende Führungsaufgaben müssten von entscheidenden Großparteien getragen werden. Analytisch kann man anfügen, dass der in der Literatur häufig prognostizierte Funktionsverlust dieser Führungsebene zu einer Veränderung des gesamten Parteiensystems der Bundesrepublik führen würde. Da die kleineren Parteien bisher weder mit Blick auf ihre innere ideologische und interessensbezogene Breite noch hinsichtlich ihres Leitungspersonals ein Substitut darstellen, strebte man auf ein Vakuum zu - sofern sich das Parteiensystem ernsthaft in diese Richtung bewegen sollte.

\section{Kanzlerwahlverein im Parteienstaat?}

Die Gefahr einer derart zentralen Stellung von Großparteien liegt darin, dass Machtstrukturen korrumpieren oder gar zu einer Oligarchisierung führen können. Auch von „Verbonzung“ ist gelegentlich die Rede. Manche, auch ein Bundespräsident von Weizsäcker, befürchteten gar einen Parteienstaat, ${ }^{8}$ in dessen Rahmen die Parteien sich die res publica zur Beute nehmen. Andere formulierten dagegen den dezenten Hinweis, dass ein Parteienstaat gerade das Bild der Bundesrepublik als funktionierende Demokratie abbildet. ${ }^{9}$ Man wird für das deutsche Parteien-

Ursachen und Konsequenzen, in: von Arnim, H.H. (Hrsg.): Systemmängel in Demokratie und Marktwirtschaft, Berlin, 2011, 153-161; Poguntke, T.: Ratlose Zwerge: sind die Volksparteien am Ende?, in: Bytzek, E./Roßteutscher, S. (Hrsg): Der unbekannte Wähler? Mythen und Fakten über das Wahlverhalten der Deutschen, Frankfurt, 2011, 133-154.

8 Vgl. zu dieser Diskussion Hennis, W.: Auf dem Weg in den Parteienstaat. Aufsätze aus vier Jahrzehnten, Stuttgart, 1998.

9 Lösche, P.: Parteienstaat in der Krise? Überlegungen nach 50 Jahren Bundesrepublik Deutschland, Reihe Gesprächskreis Geschichte, Heft 27, Friedrich-Ebert-Stiftung, Bonn, 1999 
system jedenfalls sagen können, dass sich derartige Gefahren und Klüngelstrukturen zwar nachweisen lassen, diese aber insgesamt doch von nur peripherer Bedeutung sind. Insofern ist die Geschichte der Bundesrepublik, was ihre tragenden Parteien angeht, glücklich verlaufen. Der Parteienwettbewerb sorgt im Übrigen dafür, dass allzu ausgeprägte Verkrustungen nach einer Weile erkannt, aufgebrochen und korrigiert werden, nicht zuletzt durch die Abwahl von Parteirepräsentanten aus entsprechenden Ämtern. Rheinland-Pfalz nach Bernhard Vogel ging auf diese Weise verloren, weil der eigene Übermut von den Wählern bestraft wurde. Die Rückeroberung eines einmal verlorenen Landes fällt einer Großpartei aber stets schwer. Ein Fehlverhalten des Führungspersonals hat enorme Kollateralschäden zur Folge.

Natürlich besteht auch für die CDU das Problem, als Regierungspartei ihre eigene Programmatik nicht optimal entfalten zu können. Ihr wird nachgesagt, dass sie eher ein Kanzlerwahlverein als eine wirkliche Interessen-Integrationspartei sei. Der Begriff hat aber mehr journalistische Bedeutung, wirkt ggf. eher in der Propaganda des Gegners, als wirklich ein Problem dazustellen. Ein gut arbeitender Kanzler (bzw. Kanzlerin) ist für eine Volkspartei natürlich ein erstklassiges Aushängeschild und ein Wählermagnet. Hier wird Aufmerksamkeit erzeugt, die der Oppositionspartei nicht zukommt. Die Lokomotivfunktion einer erfolgreichen Kanzlerschaft ist für die Partei nicht zu übertreffen. Das Risiko liegt im Erfolg der Kanzlerschaft. Stellt er sich nicht ein, floppt ein Kanzler Erhard oder ein Kanzler Kiesinger, so leidet die Partei mit. Kanzlerkandidaten nicht strategisch effizient ausgewählt zu haben ist ein Versehen, das der Wähler abstraft. Jede Partei in dieser Lage wünschte sich, ein Kanzler(kandidaten)wahlverein zu sein. Das Stichwort „Kanzlerwahlverein“ hat mithin ganz zu Unrecht einen schlechten Leumund, ist es doch Gold wert.

Natürlich muss eine Regierungspartei noch mehr dem Charakter der Integration entsprechen, was Abstriche bei der Identität vorzunehmen bedeutet. Insofern sind entsprechende Phasen einerseits attraktiv, weil sie Politik- und Machtinteressierte anziehen. Andererseits irritieren sie ideologisch orientierte Menschen, die ein bestimmtes Programm verwirklicht sehen möchten. Hier kommt es erneut auf die Parteiführung an, der Gesellschaft zu verstehen zu geben, dass alle diese Fragen bedacht werden. Insofern müssen von Zeit zu Zeit auch die ideologischen Flügel auf ihre Kosten kommen und öffentliche Anerkennung finden. 


\section{Die CDU in der Moderne}

Ruhige Zeiten für die Nachkriegs-CDU, turbulente Zeiten für die CDU des 21. Jahrhunderts - eine derartige Unterscheidung liegt für die so Argumentierenden mit Blick auf die Erzeugung kurzlebiger Aufmerksamkeit nahe. Die darin zum Ausdruck kommende Unkenntnis der Nachkriegszeit wird meist unbekümmert hingenommen.

Durchaus ernsthafter ist zu fragen, wie sich die CDU in der Modernität, im Säkularisierungsprozess und im Rahmen der Globalisierung behaupten kann, wie sie mit dem gesellschaftlichen Individualisierungsvorgang auskommt und wie sie der allgemein konstatierbaren Bindungsschwäche in der Gesellschaft zu entgegnen sucht. Hinzu tritt die demografische Entwicklung, die zu einer gesellschaftlichen Alterung führt und die sich v.a. in einer Alterung der Mitgliederschaft niederschlägt. Die große Herausforderung für die CDU liegt in der Frage, ob sie über die notwendige Verarbeitungskapazität verfügt - nicht nur an der Spitze, sondern im gesamten Apparat. Der Säkularisierungsprozess muss keine unüberwindliche Hürde sein, wenn man den Integrationsprozess ernst nimmt und neben konfessionell gebundenen auch kulturchristlich orientierte Mitbürger, Konfessionslose oder gar konfessionell anders geprägte Bürger, Muslime etwa, ansprechen möchte. Die CDU als eine Partei, die sich auch mit Wirtschaftsfragen identifiziert, hat den Globalisierungsprozess ihrerseits mitgetragen, steht also dem Gedanken des Freihandels näher als dem des Protektionismus, wozu auch die ökonomische Lage Deutschlands in Europa beiträgt. Insofern sind die wirtschaftlichen und kulturellen Entwicklungen nicht per se Probleme für eine Partei, die sich modern aufstellen will und kann.

\section{Bindungen, Milieus and all that}

Schwieriger ist es, der allgemeinen Bindungsschwäche zu entgehen. Dieses Thema ist in der sozialwissenschaftlichen Fachliteratur stark vertreten, wenn auch zwischenzeitlich recht redundant. Es geht um die „Milieuthese“, nach der die abschmelzenden Milieus zum Rückgang der Wählerschaft führen. Dazu kann man vielerlei ausführen. Jedenfalls müsste der Milieuschwund längst zum Abdanken der beiden Volksparteien geführt haben. Doch die Geschichte geht unbeeindruckt weiter - so dass gelegentlich eine dezente Korrektur seitens der Analytiker erbeten werden könnte.

Jedenfalls ist die Bindungsschwäche an sich vollkommen unstrittig. Hier kann man allerdings auf die nach wie vor hohe Zahl der CDU-Parteimitglieder ver- 
weisen, wobei es natürlich, wie bereits erwähnt, einen starken Überhang im Bereich der älteren Mitglieder gibt. Insofern steht die CDU hier durchaus vor einer gravierenden Herausforderung, die sie über kurz oder lang bewältigen muss. Heutzutage stellt das Werben um Mitglieder, auch angesichts der weitverbreiteten Zögerlichkeit, sich festlegen zu wollen und zu binden, eine Herausforderung dar. Hier erfolgreich zu sein, erfordert eine Anstrengung, die ohne die programmatische Intonation durch führende Parteivertreter nicht möglich wäre. Insofern ist das Problem der Bindungsschwäche mit anderen gesellschaftlichen Herausforderungen eng verbunden. Die gesellschaftliche Entwicklung bedingt mithin, dass die CDU einen Anpassungsprozess leisten muss, ohne Identität verlieren zu dürfen. An sich gehört es zum Leistungsspektrum einer Großpartei, dass sie in der Lage sein muss, einen Spagat zwischen unterschiedlichen Interessen zu vollführen. Allerdings müssen diese Interessen identifiziert und artikuliert werden. Sie müssen anerkannt sein und sie müssen als Teil des „Unionshauses“ verhandelt werden können. Durch dieses Ernstnehmen von Problemen und das Zusammenfügen unterschiedlicher Ausrichtungen könnte die Partei wieder Integrationskraft gewinnen.

Kommt allerdings der Eindruck der Beliebigkeit auf, der in der Vergangenheit durchaus angesprochen wurde, würde man zwar immer noch von einer Sammlung sprechen können. Allerdings meint Sammlung im Sinne einer Partei eine Konstellation, die, wie erwähnt, Zusammengehöriges auch zusammenführt. Die Sozialstruktur der Partei und die Abbildung unterschiedlicher Gruppierungen sind für eine Großpartei essentiell. Wäre sie nur die Partei der alten Leute, würde sie ihren Charakter, aber auch ihre Verankerung in der Bevölkerung schnell verlieren. Sie darf auch keine Partei sein, die in irgendeiner Weise mit den Interessen von Frauen nicht vereinbar ist, sie darf auch nicht eine Partei sein, die einzelne Regionen vernachlässigt - ein Problem, das etwa die Konservativen in Großbritannien mit Blick auf den Nordteil der Insel ganz deutlich kennzeichnet. Unterschiedliche Fraueninteressen $\mathrm{zu}$ integrieren, ist derzeit besonders schwer, weil unterschiedliche Lebensvorstellungen anerkannt werden müssen, wobei es in den gesellschaftlichen Debatten oft darum zu gehen scheint, ob nur eine einzige Lebensstrategie als legitim ausgegeben werden soll.

Auch eine zu stark auf das Alter oder „Erfahrung“ setzende Partei würde sich von jüngeren Anhängern oder gar Mitgliedern verabschieden müssen. Die Themenwelt jüngerer Bürger nicht abzudecken führt dazu, dass andere Parteien hier eine Nische finden, wie etwa die Piratenpartei. Der „Entjugendlichung“ unserer Gesellschaft entspricht auch ein Jugenddefizit in der Mitgliederstruktur. Dass 
sich hier ein Zukunftsproblem auftut, braucht nicht ausgeführt zu werden. In einer alternden Gesellschaft, die noch dazu ethnodiverser und „bunter“ wird, stellt dieses Altersdefizit ein zusätzliches Belastungsmoment für die Gesellschaft dar, weil dem Anspruch auf Problemverarbeitung, den die CDU als Volkspartei aufrecht erhält, nicht entsprochen wird. Wie die Besetzung von Führungsämtern wäre eine optimistisch stimmende Rekrutierung des Nachwuchses ein Erfolgssignal für die gesamte Parteiendemokratie.

Eine Volkspartei hat immer auch die Aufgabe, die Gesellschaft voranzubringen. Es kommt darauf an, dass innerhalb der Partei vorausgedacht wird und dabei gesellschaftspolitisch für die Zukunft tragfähige Konstrukte entstehen, die sich dann in der Praxis bewähren, sofern sie von den Bürgern akzeptiert werden und einen gesellschaftlichen Fortschritt bzw. eine Anpassungsfähigkeit bewirken. In einer Zeit, in der der demografische Wandel an Vehemenz zunimmt, wird diese Aufgabe noch bedeutsamer. Er verlangt eine noch größere Anstrengung, als ohnehin schon durch ökonomische und soziale Kräfte bedingt.

\section{VIII.Familien- und Sozialpolitik - und eine ausstehende Gesellschaftsanalyse}

Bekanntlich wird im Feld der Familienpolitik keineswegs nur mit Blick auf die Familien agiert, sondern es werden Gesellschaftsentwürfe transportiert und ggf. gar die Gesellschaft verändert - explizit wie implizit. Oberflächlich gesehen hat die Familienpolitik nur damit zu tun, wie Familien in der Politik berücksichtigt werden. Tatsächlich geht es aber darum, wie dieser vorpolitische Raum, das Leben in Familien und das Leben mit Kindern, gestaltet wird, wie akzeptiert werden kann, dass es unterschiedliche Lebensformen gibt und dass die ökonomische Entwicklung auch im familiären Bereich Anpassungsprozesse bedingt.

Oft wird umgekehrt gedacht, dass die Ökonomie auf die Familien Rücksicht zu nehmen hat. Gerade eine christlich orientierte Partei wird diesen Gedanken besonders herauszustellen versuchen. In der harten Realität aber sieht es so aus, dass man Kompromisse schließen muss, die die Menschen herausfordern und in deren Rahmen die Politik nur eine begrenzte Rolle spielen kann. Aber allein schon bei der Frage, wer die Kinder betreut - eigentlich ein Thema, die nur die Familien selbst etwas angeht - finden sich heftige Debatten, die einerseits ideologischer Natur sind, wenn die Kinder aus den Familien herausgenommen werden sollen, und es andererseits auch ein ökonomisches Interesse daran gibt, dass Frauen, insbesondere qualifizierte, sich eben nicht „nur“ einer häuslichen Tätig- 
keit widmen, sondern auch der Erwerbsarbeit nachgehen. Insofern gibt es im linken Spektrum ein Interesse an der Vollzeitberufstätigkeit von Müttern. Eine christliche Partei tut sich mit derartigen Zumutungen schwer, geht sie doch eigentlich davon aus, dass der Wert der Familie wichtiger ist.

Eine derartige Partei muss allerdings auch sehen, dass sich gerade aufgrund des demografischen Wandels vielfältige Anforderungen an die Familie richten, der sie nicht unbedingt und in jedem Fall gewachsen sein kann. Die CDU vermag sich also als moderne Partei zu profilieren und dem ökonomischen Fortschritt anzupassen, der auch eine linke Melodie enthält, oder sie kann sich denunziert wiederfinden, charakterisiert werden durch Begriffe wie „Herd“, „Heim“ und „Küche“ - um so ein Lebensmodell zu diskreditieren. Diese Ausführungen mögen illustrieren, wie ein harmloses Politikfeld für eine große Volkspartei zum Terrain einer gesellschaftspolitischen Auseinandersetzung wird, die dividierend wirken kann und auch innerparteilich zu Konflikten führt.

Auch in der Sozialpolitik tut sich manche Schwierigkeit auf. Einerseits vertritt die CDU den sozialen, marktwirtschaftlichen Kurs und legt mithin Wert darauf, dass nicht der Markt allein diktieren kann, was eine vernünftige Politik ist. Die Partei ist daran interessiert zu sehen, dass soziale Komponenten im marktwirtschaftlichen Prozess von Seiten des Staates beachtet werden. Genauso wie es falsch wäre, nur auf den Staat zu setzen, was dem subsidiären Denken der Union widerspräche, genauso verfehlt wäre es, sich nur dem Markt anzudienen und von ihm eine Strukturierung ganzer Politikfelder zu erwarten. Die CDU als die Erfinderin der Sozialen Marktwirtschaft bleibt dem Markt verbunden, schon weil Planwirtschaft nie funktionierte. Das Soziale war aber der Punkt, auf den es ankam. Für die CDU tut sich dabei die Frage auf, was das Soziale im 21. Jahrhundert ausmacht, ob man es noch selbst ausgestalten will oder lieber an eine höhere Ebene weiterempfiehlt. Nach dem Selbstverständnis der Partei dürfte eine solche europapolitische Ausrichtung kaum ihrer historisch gewachsenen Identität entsprechen.

Der Anspruch der Unionsanhänger lautet vielmehr, dass man den Zumutungen des Marktes, national wie europäisch und global, nicht unmittelbar ausgesetzt sein möchte, aber auch nicht ein Gesellschafts- und Wirtschaftsmodell mittragen kann, das vom Prinzip der Gleichheit bestimmt wird. Leistung muss sich lohnen, sonst kann man nicht von einer Marktwirtschaft sprechen. Auch im Globalisierungsprozess des 21. Jahrhunderts muss das sozialstaatliche Profil Deutschlands erkennbar sein und muss der von der CDU stark mitgeprägte rheinische Kapitalismus seine Fähigkeit zur Problemlösung unter Beweis stellen, selbst wenn die 
operative Politik zwischenzeitlich den bestimmenden Einzugsbereich verlassen hat.

Eine zusätzliche Schwierigkeit der sozialstaatlichen wie der wohlfahrtsstaatlichen Politik liegt angesichts des demografischen Wandels darin, eine Entwicklungsfähigkeit vorzuhalten, die durch demografische Asymmetrien eigentlich erheblich infrage gestellt wird. Einer alternden Gesellschaft ihre Entwicklungsfähigkeit zu garantieren, fällt durchaus schwer. Am Standort Deutschland festhalten zu wollen, setzt eine marktwirtschaftliche Dynamik voraus, die den Sozialstaat in Mitleidenschaft ziehen könnte. Ist das demografische Potential bzw. das Humanvermögen ${ }^{10}$ nicht mehr in ausreichendem Maß gegeben, um die wirtschaftliche Dynamik zu erhalten, stößt das sozialstaatliche Angebot an Grenzen der Machbarkeit.

Reformen im Bereich der Sozial- und Wohlfahrtsstaatlichkeit durchzuführen, ist gerade für eine Volkspartei wie die CDU ein riskantes Unterfangen, das in anderen europäischen Staaten schon zur Abwahl konservativer Regierungen führte. Das Geschick besteht also darin, die Reformfähigkeit des Landes zu erhalten und dabei seine Stabilität im Blick zu haben. Beide Vorgänge sind darüber hinaus unter Bedingungen der Parteienkonkurrenz zu sehen. Von einem begleitenden, meist dissonanten Mediensystem war noch gar nicht die Rede.

Ein allgemeines Gesellschaftsmodell zu entwickeln, auf das man sich verständigen könnte und das eine gewisse Attraktivität ausstrahlt, wäre kommunikativ von großer Bedeutung und insofern sind die Namen, die man einer bestimmten gesellschaftlichen Entwicklung gibt, durchaus von strategischer Relevanz. ${ }^{11}$ Hier ein Konzept vorzulegen, dürfte für die CDU von erstrangiger Bedeutung sein. Dies setzt eine realistische Gesellschaftsanalyse voraus. Über Vordenker zu verfügen, die eine Ahnung von gesellschaftlichen trends vorweisen können und die Tendenz einer gesellschaftlichen Entwicklung auf den Begriff zu bringen vermögen - nicht zu verwechseln mit der viel einfacheren Benennung eines Trends -, ist deshalb für beide Volksparteien sehr bedeutsam. Kleinere Parteien können es sich leisten, Nischenaspekte der gesellschaftlichen Entwicklung zu besetzen. Auch hier wird deutlich, dass das Verantwortungsspektrum einer Volkspartei im wahrsten Sinne des Wortes von tragender Bedeutung ist. Über

10 Kaufmann, F.-X.: Schrumpfende Gesellschaft. Vom Bevölkerungsrückgang und seinen Folgen, Frankfurt am Main, 2005.

11 Das war etwa mit der „Neuen Mitte“ erreicht, aber auch „New Labour“ ist Vergangenheit, s. Giddens, A.: New Labour ist tot, in: Cicero, Juni 2010, 38-41. 
eine stringente Gesellschaftsanalyse zu verfügen, die nicht aus der Addition von Daten und Fakten besteht, sondern die Dynamik der Gesellschaft ebenso erfasst wie ihre treibenden Kräfte, wäre ein Glücksgriff für jede derartige Gruppierung und für eine Partei an der Macht ein Schibboleth für den Erfolg.

\section{Die CDU im Osten}

Die Herstellung sozialer Kohäsion und gesamtgesellschaftlicher Entwicklungsfähigkeit ist sicherlich ein essentielles Moment, dem die Volkspartei CDU zu dienen hat. Allerdings treten weitere Aufgaben hinzu, wie etwa die Bewerkstelligung der nationalen Integration mit Blick auf das Verhältnis von Ost- und Westdeutschland. Im Fall der Regierungsbeteiligung gilt als Aufgabe, auch angesichts der Schwierigkeiten des demografischen Wandels keine Region zu vernachlässigen und den Aufholprozess des Ostens weiterhin zu unterstützen. Hinzu treten aber Herausforderungen organisatorischer Art. Es war die große Aufgabe der CDU nach der Wiedervereinigung, sich ihres gesamtdeutschen Charakters zu versichern und die Transformation der Ost-CDU in einen Teil der deutschlandweiten Partei zu bewerkstelligen.

Die Ost-CDU war als gesamtdeutsche fortschrittliche Kraft entstanden, etwa unter der legendären Führung von Jakob Kaiser im späteren Ost-Berlin, wo sich anfangs sogar eine „Reichsgeschäftsstelle“ formierte - ein bereits 1947 beendetes Unterfangen. Die Ost-CDU nahm dann eine Entwicklung, die man als systemtragend ansehen kann, ohne ihr zu sehr nahetreten zu wollen. ${ }^{12}$ Diese Systemaffinität konnte zwar für einzelne Mitglieder dazu führen, dass man einerseits das System unterstützte, ohne andererseits der herrschenden sozialistischen Ideologie folgen zu müssen. Insofern bot die CDU einen gewissen Freiraum, der allerdings ideologischer Natur blieb und im engeren Sinne keine systemtranszendente Funktion hatte. Diesen Sachverhalt zu überspielen, wäre unfair. Insofern bestand nach 1989/90 die Herausforderung darin, eine derartige Union, die mit der Westpartei nicht kompatibel war, zu transformieren, Personen, die damals wirkten, aber nicht zu sehr in das System integriert waren, zu gewinnen und andere, die dies eben doch waren, zurückzuweisen. Diese Leistung ${ }^{13}$ der gesamtdeutschen CDU wird zu wenig gewürdigt, obgleich sie noch 2012 darauf aufbau-

12 Fischer, A./Agethen, M.: Die CDU in der sowjetisch besetzten Zone/DDR 1945-1952, Sankt Augustin, 1994.

13 Küsters, H.-J.: Die Ära Kohl im Gespräch. Die Vereinigung von CDU (Ost) und CDU (West) 1990, in: Historisch-Politische Mitteilungen, 18 (2011), 167-192. 
en kann. Auch hier ist umgekehrt für die Gegner der CDU zu sagen, dass es diesen leicht fiel, die sogenannte Block-CDU innerhalb der gesamtdeutschen CDU ausfindig zu machen, also Instanzen in der Ost-CDU zu identifizieren, die bereits vor 1989 bestanden. Die Integrationsleistung der gesamtdeutschen CDU als Volkspartei gilt es aber herauszustreichen, weil es dabei sowohl um Integration als auch um Bereinigung ging. Dieser Prozess wurde in der Literatur bislang nur unzureichend aufgearbeitet.

Ein Weiteres kommt hinzu: Die Ost-CDU hatte ihrerseits nach dem Winter 1989/90 als Volkspartei neue Aufgaben zu übernehmen, und zwar mit Hilfe der West-CDU. Diese Aufgaben ergaben und ergeben sich aus der gesamtdeutschen Integration. Die Sanierung der SED-Hinterlassenschaft im Osten war eine gewaltige Herausforderung, die auch von der CDU im Osten getragen werden musste. Neben herausragenden Politikern gab es dabei solche, deren Profil eher vergangenheitslastig war. Insgesamt aber kann man festhalten, dass die Vergangenheit der CDU im Osten eigentlich kein Thema mehr ist - ein Integrationserfolg für sich, der weit über die Partei hinaus zu konstatieren ist und dem für die politische Kultur Deutschlands, gerade auch in der Auseinandersetzung mit der SED/PDS/Die LINKE, große Bedeutung zukommt. Das Integrationskonzept der Union war parteihistorisch ein Erfolg.

Im weiteren Rückblick ist ebenfalls erwähnenswert, dass die CDU im Osten kurioser Weise in den 1990er Jahren in die Lage kam, als die Arbeiterpartei schlechthin angesehen zu werden, einfach weil die Arbeiterschaft im Osten Deutschlands die Partei von Norbert Blüm und Helmut Kohl 1990 wie auch 1994 stark favorisierte. Zur Kuriosität der CDU zählt im Osten weiter, dass ehemalige Arbeiterhochburgen, und damit Hochburgen der Sozialdemokratie und der Kommunistischen Partei, christdemokratisch revidiert wurden. Das betrifft etwa Sachsen. Dass diese Strategie über die vergangenen beiden Jahrzehnte nicht durchgehalten werden konnte, hat mit gesamtstaatlichen Entwicklungen zu tun und führte 1998 zur Abwahl der Regierung Kohl, die innere Reformen nur unzureichend umgesetzt hatte, insofern den Gründungsprozess des Nationalstaates Deutschland 1990, der eine Wiedergründung war, nicht, wie in der BismarckZeit, mit sozialen Reformprogrammen krönte. Dafür hat Helmut Kohl mit dem Verlust der Macht bezahlt.

\section{Die Schwester im Süden}

Neben der regionalen Thematik „Ost-CDU“ gibt es noch einen weiteren Bereich, der erhebliche Herausforderungen birgt: die Fraktionsgemeinschaft von CDU 
und CSU im Deutschen Bundestag. ${ }^{14} \mathrm{Zu}$ verweisen ist hier auf die Rolle der CSU im Rahmen der Unionspolitik. Die CSU kann sich als eigenständige Partei verstehen und verfügt in Bayern über eine eindeutige und weitgehend erfolgreiche Profilierung. Die CSU ist aber nicht nur eine Regionalpartei, sondern sie erhebt seit Jahrzehnten auch große bundespolitische Ansprüche. Sie kann inhaltlich gesehen zudem als ein Korrektiv zur CDU verstanden werden, insofern sie stärker den konservativen Flügel der Unionsparteien abdeckt und auch die soziale Komponente im Parteinamen sehr ernst nimmt. Der nationale Anspruch der CSU hat sich in der bundesdeutschen Geschichte mehrfach auch in innen- wie außenpolitischen Angelegenheiten niedergeschlagen. Aus CDU-Sicht - und damit aus Sicht des stärkeren Teils des Unionsspektrums - liegt die Herausforderung der CSU darin, dass sie immer wieder als Partner ernst genommen werden will und muss, diese Berücksichtigung aber im Alltag große Probleme bereitet. Von gegnerischer Seite werden die Unionsparteien eher weniger als Einheit angesehen. Intern aber ist es so, dass die CSU eine besondere Sichtbarkeit erlangen möchte, die sie eigentlich nur auf Kosten der CDU erreichen kann. Damit ist programmiert, dass die CSU in jeder Koalitionsregierung versucht, eine spezifische Rolle zu spielen. Die dabei entstehenden Friktionen wirken auf ein Publikum, das ein eher einheitliches Auftreten der Unionsparteien erwartet, meist spalterisch und störend.

Muss man daraus folgern, dass die Existenz der CSU eine Schwäche der CDU darstellt? Auch hier kommt es auf das Führungspersonal an, ob es miteinander harmoniert oder sich subtil bekämpft. Die Situation ist sicher singulär in der europäischen Parteienlandschaft, ${ }^{15}$ wobei weniger wahrgenommen wird, dass das Spektrum der Unionsparteien durch diese Lösung, gesamthaft betrachtet, noch verbreitert wird. Es kommt also auch hier auf die Geschicklichkeit an, als Regierung unterschiedliche Interessen zu integrieren und damit unterschiedliche Identitäten gelten zu lassen. Insofern kann aus der Vielfalt im Erfolgsfall eine Stärke werden. Dass dagegen oft das Profilierungsbedürfnis der CSU-Spitze überhand nimmt, steht außer Frage, aber umgekehrt ist der input an konservativer Programmatik und an einem gewissen populistischen Realismus durch die CSU auch für die CDU ebenso konstitutiv wie konstruktiv.

14 Schwarz, H.-P. (Hrsg.): Die Fraktion als Machtfaktor. CDU/CSU im Deutschen Bundestag 1949 bis heute, München, 2009.

15 Grabow, C. (Hrsg.): Christlich-Demokratische Parteien in Westeuropa. Stand und Perspektiven, Sankt Augustin, 2012. 


\section{En Passant: Medien und Union}

Die innerparteiliche Vielfalt der CDU und ihrer Schwesterpartei trägt dazu bei, dass die Widersprüche und Friktionen, die dabei entstehen, im Mediensystem denkbar große Beachtung finden. Die Medienlandschaft überhaupt - und weniger die Medien im Allgemeinen als die Journalisten im Besonderen - stellen für konservative Parteien durchaus eine Herausforderung dar, weil es keine Unterstellung sein dürfte anzunehmen, dass der mainstream der Journalisten nicht eben konservativ eingestellt ist. Qualitativ gute Umfragen können natürlich dazu führen, dass die veröffentlichte Meinung etwas korrigiert wird; meinungsbildende Medien erweisen sich aber meist als stärker als demoskopische Befunde. Wenn wir die Medienwelt beachten, können wir auch nicht nur den unmittelbar politischen Raum wahrnehmen, sondern müssen das Mediensystem als eines sehen, das auch für sich genommen eine Bühne inszeniert, die zur Veränderung der Gesellschaft, etwa im Bereich der Werte, beiträgt. Die auf Spektakel, Zirzensik und Zuschauerquoten Wert legende Fernsehwelt produziert damit ein Weltbild, das mit dem solider Unionsanhänger nicht unbedingt kompatibel sein muss.

\section{Die europäische Finalitätsfrage}

Dass die CDU auch zum außenpolitischen Erfolg Westdeutschlands und dann auch des wiedervereinigten Deutschlands einen erheblichen Beitrag geleistet hat, wird niemand bestreiten. Gleichwohl befindet man sich in einer internationalen Politiklandschaft, die erhebliche Volatilität zum Ausdruck bringt, v.a. im elementaren Bereich der wirtschaftlichen Stabilität. Das währungspolitische Abenteuer Euro zeigt seit Jahren seine Tücken und wirft die Frage auf, ob dieses europapolitische Projekt, das maßgeblich von der CDU mitgedacht wurde und ihrem außenpolitischen Profil durchaus entspricht, wirklich eine gute Idee war. Käme zu einem crash, könnte es passieren, dass die herausgehobene Position der Kanzlerin Schaden nimmt. Die Dominanz ökonomischer Fragen priorisiert seit einigen Jahren die Europapolitik. Der „immer engere Zusammenschluss“ entspricht zwar der Integrationsphilosophie der EU, stößt aber an Grenzen, wie sie das Bundesverfassungsgericht zuletzt in seinem Lissabon-Urteil darlegte.

Insofern kommt die Frage nach der Finalität erneut zum Vorschein, die viele Europapolitiker, auch in der Union, nicht beantworten möchten. Trotzdem steht 
das Gespenst eines europäischen Bundesstaates ${ }^{16}$ vor aller Augen und die Partei Konrad Adenauers muss sich fragen lassen, wie weit sie den Integrationsprozess treiben möchte, ohne die Eigenstaatlichkeit Deutschlands aufzugeben. Wenn Art. 146 des Grundgesetzes tatsächlich in absehbarer Zeit auf die Tagesordnung der Politik kommt, ${ }^{17}$ stünde nicht nur die CDU vor einer Grundsatzentscheidung, wie sie sich seit Herrenchiemsee nicht mehr gestellt hat. Es darf davon ausgegangen werden, dass versucht würde, eine derartige Fundamentalentscheidung durch eine andere, damit verbundene Agenda zu überlagern, etwa durch einen europäischen Transformationsprozess, der dann zum Abstimmungsgegenstand gemacht würde. Wie immer man sich zu dieser Frage positioniert, sie käme einer nationalversammlungsartigen Jahrhundertentscheidung gleich.

\section{Die geostrategische Herausforderung}

Die Deutschland seitens der CDU zugedachte geopolitische Rolle zu bestimmen, ist keinesfalls einfach. Auf dem europäisch-asiatischen Großkontinent verschieben sich die Mächte. Die USA geraten in eine distanziertere Position, in der Ära Obama verloren die transatlantischen Beziehungen, auch wenn es zu einem spektakulären Auftritt der Kanzlerin im Kongress gekommen ist, klar an Bedeutung. Der Aufstieg Chinas und der Machtantritt Obamas führten in der CDU noch nicht zur Weiterentwicklung eines klaren außenpolitischen Profils. Die Union ist garantiert keine Partei, die zu einer Äquidistanz zu den USA und China neigt, aber wie in der Europapolitik stehen die Beziehungen zu China unter dem Primat ökonomischer Herausforderungen. Während der türkische Beitritt zur EU hinausgezögert werden kann, vermag man die Anerkennung Chinas kaum zu verweigern, obgleich es sich weiterhin um eine kommunistische Macht handelt. Man darf von der CDU erwarten, dass sie nach außen sowohl realpolitisch agiert als auch durch eine klare normative Agenda ihren Kurs findet. Gleichwohl kam es $2011 \mathrm{zu}$ einer außenpolitischen Isolierung Deutschlands, als der liberale Außenminister sich nicht in der Lage sah, den Libyeneinsatz Großbritanniens und Frankreichs mitzutragen. In der Konsequenz leidet die außenpolitische Kompetenz der CDU, nicht nur personell, unter mangelnder Sichtbarkeit.

16 Lübbe, H.: Vereinigte Staaten von Europa?, in: FAZ, 4. November 2011, 31.

17 „Noch mehr Europa lässt das Grundgesetz kaum zu“ (Interview mit BVerG-Präsident Andreas Vosskuhle), in: FAS, 25. September 2011, 36-37; Murswiek, D.: Der Grundsatz der souveränen Staatlichkeit als unabänderliches Verfassungsprinzip, in: Kraus, H.-C./Wolff, H.A.: Souveränitätsprobleme der Neuzeit. Freundesgabe für Helmut Quaritsch anlässlich seines 80. Geburtstages, Berlin, 2010, 95-147. 


\section{Kein Fischzug in den Ländern}

Im Rahmen einer Analyse der Lage der CDU vor der Bundestagswahl 2013 erkennt man deutliche Herausforderungen, allein schon aufgrund der prekären Wahlergebnisse der CDU auf Länderebene. ${ }^{18}$ Der Partei ist es nahezu vollständig misslungen, sich eindrucksvoll zu profilieren. $\mathrm{Zu}$ diesem Mechanismus der Abwahl trägt natürlich bei, dass in Deutschland übertrieben viel gewählt werden muss. Der überforderte Wähler nimmt die Wahlchance jeweils auch an, aber vorrangig unter dem Aspekt, das entfernte Berlin vor Ort abstrafen zu können. Insofern ist dieser Mechanismus selbstverschuldet und läuft in unterschiedlichen Konstellationen immer wieder gleich ab. Wenn sich die Deutschen einen derartigen Mechanismus leisten, wird man immer auf ähnliche Entwicklungen zurückkommen können. In den einzelnen Bundesländern muss schon viel Profil und personelle Professionalität vorhanden sein, um eine Regierungspartei diesem Abstrafungsprozess entgehen zu lassen. Die persönliche Integrität der jeweiligen Landesregierung muss außer Zweifel stehen. Das war aber bekanntlich in verschiedenen Landeshauptstädten nicht der Fall. Düsseldorf ging 2010 selbstverschuldet verloren, im Stammland Baden-Württemberg hat nicht nur Fukushima das Wahldebakel 2011 verursacht und ebenso verspielte die Parteispitze in Hamburg das Ansehen in einer schwierigen schwarz-grünen Koalition. ${ }^{19}$ In einer Anti-Berlin-Stimmung trotzdem die eigene Bastion attraktiv vorzuhalten, setzt eine Leistung voraus, die top-down erfolgen muss. Die Auswahl der Führungsgarnitur und ihre Ausstrahlung auf das Ansehen der CDU wurden bereits mehrfach erwähnt.

\section{Bedingungen des Machterhalts}

Die CDU erweist sich in der bundesdeutschen Geschichte zwar als Erfolg, kann daraus aber keineswegs eine Garantie für weiteres Reüssieren ziehen. Wenn es der Partei nicht gelingt, das Lebensgefühl der Bürger zu erfassen, und zwar aller Schichten und aller Altersklassen, fällt sie hinter ihre eigenen Möglichkeiten zurück. Markante Persönlichkeiten anzubieten, eine mobilisierende Programma-

18 Vgl. vorausgegangene Studien, etwa Kronenberg, V./Mayer, T. (Hrsg.): Volksparteien: Erfolgsmodell für die Zukunft?, Freiburg, 2009; Mayer, T.: Volksparteien - voreilige Grabgesänge?, in: Gloe, M./Reinhardt, V. (Hrsg.): Politikwissenschaft und politische Bildung, Festschrift für Udo Kempf, Wiesbaden, 2010, 59-67; ders.: Die CDU Deutschlands. Eine Volkspartei in Turbulenzen, in: Grabow, K., a.a.O., 63-76.

19 Zur Möglichkeit derartiger Bündnisse ausführlich der Band von Kronenberg, V./Weckenbrock, C. (Hrsg.): Schwarz-Grün. Die Debatte, Wiesbaden, 2011. 
tik vorzuhalten, ein klares Profil zu zeigen und zu schärfen, all dies zählt zu den Erwartungen an eine Großpartei, die täglich ihre Existenzberechtigung unter Beweis stellen muss. Zum Wesen einer catch all party zählt, dass das ideologische Profil nur von eingeschränkter Bedeutung sein darf. Die CDU als Volkspartei hat andere Ansprüche an sich anzulegen als diverse Kleinparteien, deren stetiges Anwachsen vor einiger Zeit noch erwartet wurde. ${ }^{20}$

Die CDU steht und fällt mit einer Führungsleistung, um die sie nur mit der Sozialdemokratie ringt. Dabei geht es geht nicht nur um die Führung einer Großpartei, sondern auch um die Fähigkeit, das Staatsschiff durch turbulente Gewässer zu steuern. Dass die CDU fähig ist, die dafür notwendige Führungsauslese zu betreiben, muss sie immer wieder unter Beweis stellen. Misslingt ihr dies, nimmt auch die Bundesrepublik Schaden. Insofern ist der auffallende Abgang namhafter Persönlichkeiten der Union ganz besonders bemerkenswert. Das Problem besteht auch darin, dass das Mediensystem herausragende Politiker einerseits besonders schätzt und gerne intensiv analysiert, andererseits aber auch negativ markiert, wenn dissonante Positionen in der Partei vertreten werden und ein Leser wie Zuschauer unterhaltendes Rivalisieren sich de facto oder zumindest nach Vermutung der Journalisten abzeichnet. Leitmedien wie Der Spiegel oder die BildZeitung gefallen sich ganz besonders darin, das Spitzenpersonal zu hofieren oder zu kritisieren. Hinter der hehren Kontrollfunktion der Medien stecken auch oft banale Strategien zur Aufmerksamkeitsgewinnung, die in sich legitim, aber dem politischen System zuweilen abträglich sind. Andererseits zählen diese erschwerten Umstände von Beginn an zu den Begleiterscheinungen des Regierens in der Bundesrepublik. $^{21}$

Mithin stellt sich letztlich nicht die saloppe Frage, ob die CDU pensionsreif ist, sondern immer wieder aufs neue die wesentlich ernstere Frage, ob sie regierungsfähig ist und bleibt, ob sich in ihrem Ideenhaushalt ein Programm abzeichnet, das große Wählermassen bewegt. Neben dem Programm wird bekanntlich die Frage, wofür die Kanzlerin eigentlich steht, häufig aufgeworfen. Ähnlich kann man nach dem Profil der CDU insgesamt fragen. In modernen Kommunikationsprozessen kommt es sehr darauf an, dass man Dinge anspricht, die eine

20 Zeit-Magazin: Wer steckt hinter dem Aufstieg der Grünen zur Volkspartei, 13.10.2011; Fleischhauer, $J .:$ Die Revolution frisst die Grünen, Spiegel-Online, 19. September 2011.

21 Vgl. auch die Studie von Buchhaas, D.: Die Volkspartei. Programmatische Entwicklungen der CDU 1950-1973, Düsseldorf, 1981; Langguth, G. (Hrsg.): In Verantwortung für Deutschland. 50 Jahre CDU, Köln, 1996; besonders empfehlenswert heute Walter, F./Werwath, C./ D'Antonio, O.: Die CDU. Entstehung und Verfall christdemokratischer Geschlossenheit, Baden Baden, 2011. 
Mehrheit umtreibt, dass man Dinge benennt, die in der öffentlichen Meinung vorkommen. ${ }^{22}$ Politische Kommunikation ist nicht nur in Wahlkämpfen von Bedeutung. Die Kommunikation einer regierenden Partei zu vernachlässigen bedeutete, das Ende der Macht in den Blick zu nehmen. Alles auf eine Karte zu setzen, wie 1969 zu sagen: „Auf den Kanzler kommt es an“, wäre ein va banqueSpiel. Eine Volkspartei ${ }^{23}$ ist mehr als ein Kanzlerwahlverein.

An sich müsste man, wenn die Zukunft einer Partei zu beurteilen ist, die Geschäftsstellen und Fraktionsführungen genauer betrachten. Gehen von dort Impulse aus, bekommen dort Nachwuchspersönlichkeiten eine Chance, steht es um die Partei nicht schlecht. Noch wichtiger ist allerdings die öffentliche Meinung, von der alles abhängt. Gelänge es der CDU, wieder eine Art Meinungsführerschaft zu etablieren, könnte man der Partei eine gute Zukunft attestieren. Aber eine kulturelle Hegemonie erreichten die Unionsparteien ohnehin fast nie. Sie würde voraussetzen, dass eine regierende CDU auch jenseits der Regierung als Partei sichtbar wäre, eine gewisse intellektuelle Ausstrahlung hätte, namhafte Akteure sich zu ihr bekennen würden. Gegenwärtig sieht es aber danach aus, als ob die Kanzlerin allein auf weiter Flur das Feld bestreitet. Ob ihre internationale Navigationsleistung, die viel Anerkennung findet, so stark bewertet wird, dass sie ihre Partei mitzieht, muss bezweifelt werden. Deshalb sind die Aussichten, was die Bundestagswahl 2013 angeht, eher begrenzt. Die CDU bleibt stärkste Kraft, sie bleibt eine große Volkspartei, sie hält weiterhin eine hohe Mitgliederzahl, sie dürfte weiterhin - wie die SPD auch, aber im Unterschied zu Kleinparteien - Erststimmen für sich verwertbar machen. Dramatische Mobilisierungsthemen aber, die zudem noch polarisieren, zeichnen sich nicht ab. Eine Konkurrenz im Mitte-Rechts-Lager, wie etwa in Frankreich, findet sich ebenfalls nicht, auch kein verlässlicher Verbündeter. ${ }^{24}$ Das Sich-Zerstreiten und Rivalisieren der bürgerlichen Parteien aus CDU, CSU und FDP untereinander zeitigte seit 2009/10 ein phänomenales Fiasko, von dem sich die FDP kaum erholen konnte. Es stellt sich in der Folge keine dringende Notwendigkeit, dieses Lager zu wählen. Das image wurde beschädigt. Stammwähler fühlen sich kaum motiviert, wie bereits die Landtags-Wahlergebnisse dokumentierten. Diese Lethargie müsste

22 Schmid, U.: Die Lust an der politischen Schweinegrippe. Der extrem breite politische Konsens in Deutschland fördert Hysterisierung, Tabuisierung und Verdrängung zentraler Themen, in: NZZ, 25. September 2010, 17.

23 Wiesendahl, E.: Volksparteien. Aufstieg, Krise, Zukunft, Opladen, 2011.

24 Oder doch? Vgl. Dittberner, J.: Der programmierte Abstieg? Perspektiven der FDP, in: Jesse, E./Sturm, R. (Hrsg.): „Superwahljahr“ 2011 und die Folgen, Baden-Baden, 2012, 89-108. 
durch programmatische, mobilisierende Aussagen der Partei bewältigt werden: ein strategisch-programmatisches Versprechen, eine Vision: ${ }^{25}$ Derartiges wäre gefragt. Nach allen Regeln der politischen Kommunikation bedarf es zur Implantierung solcher Ansätze aber eines zeitlichen Vorlaufs. Vielleicht ist die CDU ja doch pensionsreif, wenn sie dazu nicht die Kraft aufbringt.

25 Vgl. zu strategischem Denken: Forschungsjournal „Neue Soziale Bewegungen“, 1 (2010), das sich den Volksparteien zuwendet. 